\title{
„Unsere täglichen Besuche gib uns heute ...“ Krankenhausseelsorge und Besuchsverbot
}

\author{
Gerhard Hundsdorfer
}

BESUCHSVERBOT - Ein Wort, in großen Buchstaben und fetter Schrift geschrieben und an jeder Zimmertür zu lesen, gehört seit Ausbruch der Corona-Pandemie lange Zeit zum Krankenhausalltag wie Bereichskleidung, FFP-Maske und Einmalhandschuhe.

Freundlich formuliert, aber doch konsequent gehandhabt in der Umsetzung, gehört diese Maßnahme zu den erstgereihten, weil probatesten Mitteln im Kampf gegen die Ausbreitung des Virus im Krankenhaus. Der Schutz von Patientinnen und Patienten sowie von Krankenhausmitarbeiterinnen und -mitarbeitern rechtfertigte diese Grenzziehung, weswegen sie auch von den Betroffenen größtenteils aus Vernunftgründen eingesehen und befolgt wurde.

Trotzdem meldete sich in der emotionalen Befindlichkeit Widerstand, setzte diese Maßnahme doch etwas aus, das für einen guten, menschlich stimmig gestalteten Krankenhausaufenthalt bis dahin ebenso wichtig wie unverzichtbar schien: die Möglichkeit, vertraute Menschen zu sehen, sich persönlich nach dem Befinden seiner Lieben zu erkundigen, den Krankenhausalltag mit einem Gespräch über Gott und die Welt zu unterbrechen und nicht zuletzt sich leibhaftig von einem sterbenden Menschen verabschieden zu können.

\section{1. „Ich bitte Sie, Ihre Regelbesuche einzustellen. Standard ist nicht in der Krise!"}

In eben dieser Spannung von Einsicht und Widerstand bewegten sich in diesen Tagen Krankenhausseelsorgerinnen und -seelsorger. Eine wesentliche Aufgabe ihres täglichen Dienstes war infolge des Besuchsverbots zumindest eingeschränkt, wenn nicht gar verunmöglicht. Denn in nicht wenigen Kliniken stuften Krisenstäbe Seelsorgebesuche mit Blick auf die Gesamtsituation weniger hilfreich als vielmehr riskant ein. Frick fasst pointiert zusammen: 
[...] Seelsorge und Psychotherapie im klinischen Kontext müssen sich fragen (lassen), ob sie wirklich notwendig sind. Es kommt zur Abwägung zwischen Hygiene und Prävention einerseits und dem psychosozio-spirituellen Aspekt andererseits. Die somatische, materielle Dimension ist so dominant, dass für die spirituelle kein Platz zu sein scheint. Wird auch Spiritual Care aufgeschoben auf bessere Zeiten, wie abgesagte Kongresse, Konzerte, Gottesdienste? ${ }^{1}$

Am Uniklinikum Salzburg, dem zentralen Behandlungsort für an Covid-19 Erkrankte im Bundesland Salzburg mit einer Kapazität von 108 Intensivbehandlungsplätzen und 199 stationären Normalbetten, erhielt das Seelsorgeteam in der ersten Woche im Krisenmodus auf Anfrage vom Ärztlichen Direktor entsprechend die Auskunft: „Ich bitte Sie, Ihre Regelbesuche einzustellen. Standard ist nicht in der Krise!“ Konkret bedeutete dies: Weder im Non-Covid-Bereich noch im Covid-Bereich sollte Seelsorge die Möglichkeit bekommen, ihren Auftrag zu erfüllen, kranke Menschen nicht alleine zu lassen, ihnen unmittelbare Zuwendung zu schenken und den Betroffenen so auch die bleibende Zuwendung Gottes zu versichern. Es zeigte sich in diesen Tagen ein Alltag mit konträren Facetten:

- der pastorale Auftrag zum Krankenbesuch im Sinne Jesu („Ich war krank und ihr habt mich besucht." ) - die Botschaft des von medizinischer Logik dominierten Covid-19-Krisenstabes: „Standardseelsorgebesuche sind nicht möglich.“

- die Empfehlung des Covid-19-Krisenstabes der Erzdiözese Salzburg, \#trotzdemnah zu sein - die Wahrung der Loyalität zu den Bestimmungen im Krankenhaus

- an den Schreibtisch gebundene Kolleginnen und Kollegen, die abwarten und schließlich nach alternativen Möglichkeiten der Kontaktaufnahme suchen müssen - der Wunsch der Mitarbeiterinnen und Mitarbeiter der Pflege, die Seelsorge solle die Patientinnen und Patienten persönlich besuchen, denn es sei wichtiger denn je

Deutlich wird in diesem Krisenszenario, für das es keinen vergleichbaren Erfahrungshintergrund gibt, dass Krankenhausseelsorge „prinzipiell in einem ,Zwischen'-Raum angesiedelt [ist] “2. Markant und scharf zeigen sich die Spannungsfelder, in denen Krankenhausseelsorge arbeitet.

1 Frick, Corona - eine spirituelle Erfahrung?, 205 (Hervorh. im Original).

2 Klessmann, Einleitung: Seelsorge in der Institution „Krankenhaus", 14. Zur Diskussion dieses Leitbildes: Vgl. Roser, Seelsorge im Krankenhaus, 16-20. 
Zwei davon sind im Besonderen berührt: „Seelsorge zwischen Kirche und Krankenhaus“ und "Seelsorge zwischen gesicherter Rechtsstellung und struktureller Bedeutungslosigkeit“3. Krankenhausseelsorge bezieht ihre Identität sowohl von der Kirche als auch aus dem Krankenhaus; infolgedessen hat sie sich mit zwei sehr unterschiedlichen Erwartungshorizonten auseinanderzusetzen. Und sie verfügt darüber hinaus über eine gesicherte Rechtsstellung. Tatsächlich scheint sie aber in der aktuellen Lage kaum Bedeutung zu haben, insofern die personale Präsenz am Krankenbett zugunsten anderer distanzierterer Kontaktmöglichkeiten (z. B. telefonischer Seelsorge) verzichtbar erscheint.

Nachdem „es eine wichtige Aufgabe derer [ist], die diese Arbeit tun, die Spannungen und Ambivalenzen, die sich daraus ergeben, auszuhalten und kreativ fruchtbar zu machen statt sie einseitig aufzulösen"4, muss das Besuchsverbot hinterfragt und auf mögliche Lockerungen hin geprüft werden. Dieses Vorhaben stellt freilich eine Gratwanderung dar: Systemsensibilität und das selbstbewusste Einbringen der eigenen Professionalität sind in Einklang zu bringen. (In den Alltag übersetzt: Zur medizinischen Bewältigung der Krise wurde die gesamte Klinikorganisation verändert, Hygiene- und Behandlungskonzepte mussten erarbeitet werden, ständig änderten sich Leitfäden und Richtlinien oder wurden neu erlassen, die Mitarbeiterinnen und Mitarbeiter waren entsprechend verunsichert, Abläufe in Medizin und Pflege mussten neu aufeinander abgestimmt werden u.v.m. In dieser Situation galt es, bestmöglich, mit aller Kraft und allen Möglichkeiten, aber auch mit aller Sensibilität und Behutsamkeit sowie Umsicht den seelsorglichen Dienst anzubieten und gegebenenfalls zu vollziehen.)

\section{2. „Wir wollen nicht unmenschlich sein. Besuche im Non-Covid-Bereich sind möglich.“}

Mit Blick auf die Rückmeldung des Pflegepersonals und auf den unersetzbaren Beitrag der Seelsorge im Kontext der Begleitung von kranken und sterbenden Menschen wurde eine Woche später eine neuerliche Anfrage beim Ärztlichen Direktor vorgenommen. „Wenn Sie es stemmen können, dann führen Sie Besuche im Non-Covid-Bereich durch. Wir wollen trotz allem nicht unmenschlich sein“, so dessen Fazit nach Bewertung der Lage.

3 Vgl. Klessmann, Einleitung: Seelsorge in der Institution „Krankenhaus“, 15-16.

4 Klessmann, Einleitung: Seelsorge in der Institution „Krankenhaus“, 14. 
Die Geschichte von Frau B. zeigt exemplarisch die Wichtigkeit und die positiven Effekte der Gewährung des persönlichen Zugangs zu Betroffenen auf:

Frau B. ist 73 Jahre alt, chronisch herzkrank und mir als Seelsorger von früheren Krankenhausaufenthalten bekannt. Sie musste nun doch wieder ins Krankenhaus gebracht werden. Eigentlich sollte ihr das erspart bleiben. Ihre prekäre körperliche Verfassung ließ der Hausärztin wohl keine andere Wabl. Der Preis: die Trennung von der geliebten Familie. Und nun? Besuchsverbot. Die Patientin selbst ist zu müde zum Telefonieren. Der Ausweg? „Könnte nicht die Seelsorge einspringen?!", fragt die Tochter an. Gesagt, getan. Täglich. Es verschärft sich zunehmend der Eindruck, was die Patientin selbst klar in Worte fasst: „Ich möchte meine Kräfte schweben lassen. Ich kann nicht mehr, ich will sterben. Sagen Sie meiner Familie, dass ich sie lieb habe. "Nach jedem Besuch rufe ich die Tochter an: Ich erzähle von meinen Eindrücken. Sie hört. Ich richte Grüße aus und übermittle Botschaften der Mutter. Sie schweigt. Sie ringt mit den Tränen. „Ja, ich spüre es auch, meine Mutter wird das nicht mehr überleben. Sie darf gehen. Und wenn es soweit ist, dann dürfen wir auch zu ihr, hat die Ärztin versprochen. "Am Ende eines jeden Telefonats: Dankbarkeit, dass sie wenigstens über die Seelsorge ihrer Mutter nahe sein kann. Schließlich arrangiere ich ein Telefonat zwischen Mutter und Tochter. Ich wähle, gebe der Patientin das Telefon, ein freudiges „Hallo, wie geht's dir?" Die Tochter fällt in einen Redefluss, spricht unentwegt. So viel hat sie ibr noch zu sagen. "Wir passen auf Vati auf. Mach dir keine Sorgen, du darfst loslassen!" - und anderes mehr. Die Patientin hört zu, ein schwaches "Ja!" als Antwort, dann schläft sie ein. Die Tochter merkt es nicht. Redet weiter. Erst als ich das Telefon nehme und ihr das sage, bricht ibr Reden ab. Was bleibt: "Schön, dass ich meine Mutter noch einmal gehört habe! Danke!" - Frau B. stirbt eine Woche später. Ibre Tochter erhält eine Ausnahmegenehmigung und darf die letzten beiden Tage ganztags bei ihr sein, Nächtigung inklusive. Bei meinem Besuch eine Stunde vor dem Tod der Patientin bedankt sich die Tochter nochmals für meine Besuche und die Telefonate. Es sei so wichtig für sie gewesen, unabhängig von ärztlicher Auskunft zu erfahren, wie es ibrer Mutter wirklich geht, wie sie in der persönlichen Begegnung erlebt wird.

Eine skizzenhafte Analyse des Begleitprozesses verdeutlicht die Folgen des Besuchsverbots für Patientin und Angehörige, und zeigt zugleich auf, was Seelsorge als Stellvertretung aufzufangen vermag. Frau B. ist durch den Krankenhausaufenthalt sozial isoliert, ihr fehlen in den letzten Lebenstagen, in der Phase ihres Loslassens, die Vertrautheit und Geborgenheit der häuslichen Umgebung. Verschärft wird diese Entfremdung durch das Aus- 
bleiben ihrer nächsten Angehörigen, die emotionales Getragensein, Wärme, Liebe und Unterstützung vermitteln könnten. Die Angehörigen können diesem Auftrag, den sie auch für sich selbst erfüllen wollen, nicht nachkommen. Sie sind zudem in einem hohen Maße selbst unterstützungsbedürftig. Ihnen bleiben das Erleben von Schmerz und Leid sowie das Begleiten des Weges des Abschiednehmens vom nahestehenden Menschen (zunächst und über weite Strecken) verwehrt. Die Entscheidungssituation, ob noch weitere lebenserhaltende Therapien vollzogen werden sollen, können sie nur fernmündlich begleiten. Aus dieser Distanz heraus müssen sie sich auf die medizinische Kommunikationskultur einstellen, die einen ganz spezifischen Blickwinkel auf Körper und Krankheit ins Wort fasst. Der persönliche Blick auf die Empfindungen der Patientin sowie die dialogische Rücksprache mit ihr, was Angehörige als Sprachrohr für sie vermitteln sollen, sind nicht möglich.

Der Seelsorger ist in dieser Situation Kontaktperson und als solcher Stellvertreter der Angehörigen und Brückenbauer zwischen Patientin und Tochter. Seine Präsenz am Krankenbett ermöglicht personale Begegnung, die Telefonate einen mitmenschlichen Austausch. In beide Richtungen geschieht emotionale Entlastung durch die im Seelsorger vermittelte Unmittelbarkeit. Zusätzlich zu den medizinischen Fakten und der daraus resultierenden finalen Lebenssituation spielt die „Dimension der Gefühlsarbeit" ${ }^{\text {" }}$ eine wichtige Rolle; Trauerarbeit kann dadurch begleitet werden.

Mit einem Wort: Seelsorge leistet einen unersetzbaren und speziellen Beitrag im Begehen und in der Bewältigung dieses Prozesses, dem weder Ärzteschaft noch Pflegekräfte in dieser Intensität nachkommen können. In der personalen, direkten Begegnung schafft sie einen Raum, der Erleben, Erzählen und dialogischen, wenn man so will, auch trialogischen Austausch ermöglicht. Seelsorge erweist sich im Kontext des Besuchsverbots aufgrund ihrer kommunikativen Möglichkeiten als unverzichtbar. ${ }^{6}$

Mit der generellen Besuchserlaubnis ist zwar der Weg zur Wiederaufnahme des regulären Seelsorgedienstes am Krankenbett geebnet, in der konkreten Umsetzung gewinnt dann allerdings eine grundlegende Fragestellung an Bedeutung: Welches Risiko gehen Seelsorgerinnen und Seelsorger mit Besuchen am Krankenbett ein, wie gehen sie mit ihren eigenen Sorgen, mitunter auch Ängsten um? Die Ausgangslage ist eindeutig: Im Krankenhaus ist die Gefahr der Ansteckung Dritter durch Seelsorgerinnen und Seelsorger wie auch die Gefahr der Eigenansteckung erstens höher

5 Klessmann, Einleitung: Seelsorge in der Institution „Krankenhaus“, 18.

6 Vgl. Roser, Krankenhausseelsorge und Spiritual Care, 230-231. 
und zweitens in ihren möglichen Auswirkungen noch folgenreicher und einschneidender. Die Antwort auf dieses Dilemma hingegen fällt verschieden aus: Objektive Kriterien, wie z.B. die Verringerung der Gefährdung durch die Einhaltung entsprechender hygienischer Maßnahmen, stehen im Widerstreit mit subjektiven Risikobewertungen und Gefühlslagen. Die teaminterne Diskussion wird kontrovers geführt. Es zeigt sich die Spannung zwischen zwei Polen: die kirchliche Sendung, der seelsorgliche Auftrag, der aus dem Evangelium abgeleitete Anspruch zur Hingabe auf der einen Seite; die Selbstfürsorge sowie die Pflicht zum Schutz nahestehender Angehöriger, die womöglich auch einer Risikogruppe angehören, auf der anderen Seite. Entscheidend dabei scheint im Sinne der Unterscheidung der Geister der Blick auf die Frage zu sein, wohin die eigene mit Sorge verbundene Risikobewertung führt bzw. wohin die offensive Bereitschaft zum bedingungslosen Dienst. Eine Gewissensentscheidung wird den Seelsorgenden in jedem Fall abverlangt. Letztlich sind Sorgen, Befürchtungen und Ängste sehr ernst zu nehmen und sie können im Entscheidungsfall ausschlaggebend für ein Nein sein; niemand soll gezwungen werden. Um dennoch das Vertrauen zu stärken, das Sicherheitsgefühl zu erhöhen und dem de facto bestehenden erhöhten Ansteckungsrisiko angemessen zu begegnen, sind Informationen zur Hygiene und einschlägige Schulungen unverzichtbar. Nur so kann ein verantwortlich gestalteter Seelsorgedienst gewährleistet sein. Die konsequente Einhaltung dieser Regelungen wird in Verbindung mit mutiger, nicht zuletzt auf Gottvertrauen beruhender Hingabebereitschaft die Ausübung von Seelsorge auch unter diesen besonderen Umständen ermöglichen.

\section{3. „Es ist gut, wenn es auch im Covid-Bereich Seelsorge gibt!“}

Die Erwägungen und Entscheidungen zur Frage von Eigenschutz und Sendung intensivieren sich, wenn der Auftrag zur seelsorglichen Begleitung von an Covid-19 Erkrankten gesucht bzw. konkret wahrgenommen wird. Aus dem Vorbild Jesu, der nach biblischem Zeugnis beispielsweise die direkte Begegnung mit an Aussatz leidenden Menschen suchte, ergibt sich alternativlos der Anspruch an die Krankenhausseelsorge, auch für infektiöse Patientinnen und Patienten Ansprechperson zu sein. Dementsprechend erfolgte ein drittes und letztes Gespräch mit dem Ärztlichen Direktor des Uniklinikums, um die diesbezüglichen Möglichkeiten auszuloten. Die Erlaubnis kam ebenso prompt wie überraschend: „Analog zu Konsiliardiensten dürfen Seelsorgerinnen und Seelsorger auf Anforderung im CovidBereich arbeiten - unter strikter Einhaltung der Hygienevorschriften, was 
persönliche Schutzausrüstung und das An- und Auskleiden betrifft. Letztere Maßnahme stellt nachfolgende Besuchsmöglichkeiten im Non-CovidBereich sicher. Es ist gut und wichtig, wenn Seelsorge im Covid-Bereich präsent ist und die Patienten Begleitung finden! “7

In vielen Handreichungen für Seelsorgerinnen und Seelsorger ${ }^{8}$ wird mit gutem Grund auf die Nutzung von technischen Möglichkeiten (Telefon, Videotelefonie) verwiesen, um in Kontakt mit Covid-19-Patientinnen und -Patienten treten zu können. Die Erlaubnis zum persönlichen Besuch eröffnet indes weitreichendere Möglichkeiten in der Begleitung der isolierten, äußerst belasteten und schwer kranken Menschen; sie erweitert die Optionen in der Begleitung von Angehörigen, weil der direkte Zugang tiefere Weisen von Stellvertretung bietet. Zudem ergibt sich daraus ein $\mathrm{Zu}$ sammentreffen und ein gemeinsames Arbeiten mit dem medizinischen, dem pflegerischen und anderweitigem therapeutischen Personal, was eine unverzichtbare Grundlage adäquater Seelsorge für Krankenhausmitarbeiterinnen und -mitarbeiter darstellt. Die Einschränkung „auf Anforderung“ trägt der priorisierten Zuteilung von persönlicher Schutzausrüstung für Mediziner und Pflegekräfte Rechnung und der Hinweis auf Hygienerichtlinien setzt klare Rahmenbedingungen, die größtmögliche Sicherheit in alle Richtungen bieten.

Es ist allerdings ein eigenartiges Gefühl, die Covid-Klinik erstmalig zu betreten. In der ersten Wahrnehmung erweist sie sich mit all den baulichen Vorkehrungen und hygienischen Reglementierungen als Festung. Zugangsberechtigter werde ich wegen des freigeschalteten Dienstausweises; ausgestattet mit eigener Bereichskleidung und weiterer Schutzausrüstung erlebe ich mich als Teil des Teams. Anspannung liegt in der Luft, Unbehagen und Verunsicherung melden sich. Die Konzentration auf den eigenen Auftrag sowie die mitmenschliche Begegnung mit überlebenden, sterbenden und verstorbenen Patientinnen und Patienten bringen Sicherheit zurück, ich befinde mich auf vertrautem Terrain.

Nach Bekanntgabe der Erlaubnis für Seelsorgebesuche auf allen CovidStationen kam es zunächst nur vereinzelt zu Anforderungen, diese Einsätze auf Intensiv- wie auf Normalstationen gestalten sich jedoch fachlich anspruchsvoll und menschlich berührend. Beispielhaft hierfür soll die Beglei-

7 Ich danke dem Ärztlichen Direktor Prof. Dr. Jürgen Koehler, MA, sehr für seine Wertschätzung und für die schrittweise Eröffnung der weitreichenden Besuchsmöglichkeiten, verbunden mit der Einsicht in die Wichtigkeit seelsorglicher Begleitung gerade auch von infektiösen Patientinnen und Patienten.

8 Vgl. Roser et al., Die Corona-Pandemie als Herausforderung für Spiritual Care, 2. 
tung einer Patientin vorgestellt werden, die im Folgenden (summarisch zusammengefasst) beschrieben wird:

Die 69-jährige Frau K. hat in den letzten Märztagen 2020 das positive Testergebnis erhalten und verbringt nun schon mehrere Wochen auf der CovidNormalstation. Die Symptome der Erkrankung, die nicht allzu stark ausgeprägt waren, sind abgeklungen, als sich ein besonders zäher Kampf einstellt. Denn zur Entlassung sind zwei aufeinanderfolgende negative Testergebnisse erforderlich. Bei Frau K. wechseln aber negatives und positives Testergebnis zum wiederholten Male einander ab. Die Physiotherapeutin wird auf die innere Not der Patientin aufmerksam, fragt behutsam nach und bringt in Erfahrung, dass seelsorgliche Begleitung als hilfreich erlebt werden würde. Ärztlicherseits wird der Erfüllung dieses Wunsches zugestimmt, die zuständige Pflegeschwester übernimmt die Kontaktaufnahme mit mir. Damit beginnt ein seelsorglicher Begleitprozess, der mehrere Wochen andauert und tägliche Besuche beinhaltet. Die spirituelle Hilfe für die Patientin bleibt im Blick des Behandlungsteams. So erkundigen sich die diensthabenden Ärzte, ob der Seelsorger zu Besuch kommt. Nahezu ritualisiert sprechen wir über die Befunde, welche Hoffnung, Enttäuschung und ein immer wieder neues Ringen um Geduld und Aushalten herausfordern. Die Sorge um die Familie, vor allem um den Ehemann, der zur Covid-Hochrisikogruppe gehört, wächst immer weiter an. Wie kann sie mit ihm gut im Gespräch bleiben? Wie kann sie ihm helfen? Was darf sie ihm auch zutrauen? - All diese Fragen schwirren ihr im Kopf herum. Auch die Gottesfrage wirft die gläubige Frau auf. „Was will mir Gott damit zeigen, was will er mich lehren?", so die Stoßrichtung ihrer Überlegungen. Lichtblicke sind für sie die mitmenschliche Zuwendung des Klinikpersonals und die alles überbietende Geburt der Urenkelin, die sie aufgrund ibrer Isolation nicht mit ihrer Familie feiern kann. Fotos der Neugeborenen geben ibr Anteil an diesem freudigen Ereignis. Zum Abschluss eines jeden Besuchs beten wir gemeinsam, z. B.: "Gott, bei Dir ist das Licht, Du vergisst mich nicht ..." .

Für mich als Seelsorger sind folgende Gedanken des Jesuitenpaters Karl Kern leitend:

Wir sind zum stillen Gebet angehalten. Stille, Einkehr, Ernstnehmen der Verborgenheit Gottes sind angesagt. Gottes Zuwendung, sein

9 Vgl. Taizé-Lied: „Aber du weißt den Weg für mich“, in: Ateliers et Presses de Taizé, Chants de Taizé, 139. 
Wort begeben sich ins Schweigen. Sich im Schweigen vor Gott auszuhalten, ist Karsamstagsgebet. Aus dem Schweigen heraus können wir aussprechen, was uns bedrängt, wie Jesus am Ölberg. Keiner muss tapferer sein als der Herr selbst. Bei allem aktiven Zugehen auf Gott heißt es jedoch noch mehr, sich passiv in seinem Schmerz Gott zu überlassen. Nicht in Ärger, Unmut oder Anklage sich verfangen und hängen bleiben. ${ }^{10}$

Zwei Aspekte sollen zur Analyse herausgehoben werden:

Dieser Begleitprozess verdeutlicht die Möglichkeiten eines direkten Kontaktes im Krankenzimmer (im Vergleich zu einem Telefonat bzw. zur Videotelefonie). Trotz des obligatorischen Schutzabstandes, der zwischen den Gesprächspartnern eine räumliche Distanz schafft, kann sich eine tiefere innere Nähe zwischen ihnen entwickeln. Die Patientin wird nämlich nicht nur auf ihren Körper bzw. auf dessen Infektiosität reduziert (Abstand), sondern die unmittelbare Gegenwart des Seelsorgers lässt einen Raum für eine persönliche Begegnung entstehen, in der die Patientin sich von innen her öffnen kann (Nähe). Dies wird wesentlich dadurch ermöglicht, dass der Seelsorger einerseits ihre in Worte gefassten Gedanken hört, sie andererseits aber auch in ihrem leiblichen Ausdruck (Blicke, Bewegungen und Gebärden) wahrnimmt und schließlich zu beidem in Resonanz tritt. Mit anderen Worten: Es bedarf der Präsenz, der spürbaren Anwesenheit des Seelsorgers als Voraussetzung für das Sich-Öffnen der Patientin und damit für eine neue Qualität der Begegnung. Denn „wenn der Mensch sich von innen her öffnet, dann ereilt ihn die Begegnung wie eine Berührung auf der Haut"11. Für die „spirituelle Verarbeitung“ der belastenden Krankheitssituation spielt das Gebet, der ritualisierte Abschluss der täglichen Begegnungen, eine wichtige Rolle. Als Trialog mit Gott fasst es in Sprache, was sich in der Begegnung gezeigt hat. Es will Gott das abschließende Wort geben und damit den Blick auf seine Gegenwart und seine heilend-verwandelnde Kraft weiten. Oft wurde das vom Seelsorger gesprochene Gebet durch ein Nicken der Patientin bestätigt. Für sie ist es der Höhepunkt des Besuchs, der alles auf den Punkt bringt und in den Mittelpunkt ihres Lebens rückt, der für sie Gott ist. Das lässt den Schluss zu, dass die unmittelbare Begegnung von Angesicht zu Angesicht auch die Möglichkeit gemeinsamen Betens als „Resonanzereignis“12 schafft, das auf seine

10 Kern, Mittwoch der Karwoche, 2.

11 Vasseur/Bündgens, Spiritualität der Wahrnehmung, 267.

12 Peng-Keller, Gebet als Resonanzereignis, 7. 
Weise Kraft spendet, zum Durchhalten ermutigt und Perspektiven für die nächsten (inneren) Schritte eröffnet.

Dieses Beispiel zeigt darüber hinaus, wie sehr die gemeinsame Aufmerksamkeit des Behandlungsteams auf innere, spirituelle Bedürfnisse der Patientin „not-wendig“ sein kann - und wie das seelsorgliche Gespräch von Angesicht zu Angesicht möglicherweise den medizinisch dominierten, dazu noch infektiösen Raum zu verwandeln vermag.

Seelsorge ist so etwas wie eine Heterotopie. Sie ist bewusster Partner gemeinsamer Spiritual Care [...]. [...] Spiritual Care eröffnet gemeinsam mit der Seelsorge einen unbestimmten Raum für Spirituelles. [...] Seelsorge besiedelt diesen offenen, manchmal unbestimmten Raum und macht ihn zu einem, Anderort $[$ [...]. [...] Christliche Seelsorge trägt auf diese Art und Weise bei zu einer menschenfreundlichen Kultur, gründend in einer menschenfreundlichen Haltung [...]. ${ }^{13}$

\section{Resümee}

Vor dem Hintergrund dieser theologischen Deutung der Bedeutung von Krankenhausseelsorge lassen sich folgende Aspekte als Resümee der bisherigen Erfahrungen der Seelsorge am Uniklinikum Salzburg in Zeiten der Corona-Pandemie zusammenfassen:

Wie im gewöhnlichen Klinikbetrieb, in diesen Krisenzeiten aber besonders verdichtet, basiert Heilung auch auf der zwischenmenschlichen Begegnung. Gerade die isolierten, schwer kranken, verängstigten und einer anspannenden Geduldsprobe ausgesetzten Patientinnen und Patienten sowie deren Angehörige bedürfen des Innehaltens des Behandlungsteams. Alle Mitarbeiterinnen und Mitarbeiter müssen sich vom Da-Sein dieser Menschen jenseits ihrer Infektiosität und der damit verbundenen Risiken ansprechen lassen. Wichtig ist die persönliche Komponente - sie verlangt Nähe, emotionale Wärme und einfühlsames Verstehen. ${ }^{14}$

Krankenhausseelsorge leistet in dieser Hinsicht einen unverzichtbaren Beitrag, sind ihr diese Momente der Patienten- und Angehörigenbegleitung doch als oberste Maximen ins Stammbuch geschrieben. Deutlich wird, dass dieser Behandlungsbeitrag in die Güterabwägung einbezogen werden muss, wenn es um die Einschätzung geht, ob Seelsorge mit ihrem Besuchsauftrag ein zusätzliches, womöglich größeres Risiko der Weiterga-

13 Roser, Krankenhausseelsorge und Spiritual Care, 232.

14 Vgl. Maio, Den kranken Menschen verstehen, 188-208. 
be der Infektion im Krankenhaus darstellt (auch im Vergleich mit den übrigen Gliedern des Behandlungsteams). Einen interessanten Gedanken führt in diesem Zusammenhang Frick ein: Das englische Wort „to contain“ bedeutet nicht nur „eindämmen“, sondern auch „enthalten“. Dem Psychoanalytiker Bion zufolge meint dies das Anbieten eines heilenden Gefäßes, die Verwandlung von toxischem, zerstörerischem Leid in Sinn und Bedeutung. Damit ist eine spirituelle Dimension mitten in der materiellen angesprochen und der spirituelle Kern der Sorge. ${ }^{15}$

Unbestritten ist ebenso, dass Seelsorge sich als Teil des Systems Krankenhaus verstehen muss, sich damit an alle geltenden (Hygiene-)Regelungen halten und sich ins therapeutische Konzept einfügen wird. „Seelsorge funktioniert nicht mehr als Einzelgänger-Dasein“16, wie Roser treffend feststellt.

Das für Krankenhausseelsorgerinnen und -seelsorger in vielen Kliniken ausgesprochene Besuchsverbot scheint grundsätzlich der Frage würdig und diskussionspflichtig zu sein. Die Fragestellung lautet: Was ist nun besser? Seelsorge aus Sicherheitsgründen in ihrer Dienstausübung einzuschränken, sie gar daran zu hindern? Oder aber Seelsorgende nach vorheriger eingehender (hygienischer) Unterweisung zu einer verantwortungsvollen Dienstausübung zu befähigen, damit ihr Potential hinsichtlich der mitmenschlichen Begleitung von Patientinnen und Patienten sowie deren Angehörigen genutzt werden kann - auch im Interesse einer Klinik?

„Es gibt einen Ort jenseits von richtig und falsch. Dort treffen wir uns." ${ }^{17}$ Dieser Satz wird dem mittelalterlichen Sufi Mewlana Rumi zugeschrieben und dient dem Jesuitenpater Klaus Mertes als Grundlage seiner Überlegungen zur Diskussionskultur über Richtig oder Falsch in diesen Zeiten. Er kommt zu dem Schluss:

Was geistlich richtig ist, wird sich in Corona-Zeiten nicht in der Haltung des Rechthabens und Besserwissens finden lassen, sondern jenseits von richtig und falsch durch Empathie, durch Verantwortungsbewusstsein und - sagen wir es ruhig - durch Gebet. ${ }^{18}$

Das heißt für die Krankenhausseelsorge und deren Verantwortungsträger in den Teams und auf diözesaner Ebene, im Sinne einer kritisch-loyalen Haltung das Gespräch mit den Verantwortungsträgern in den Kliniken zu

15 Vgl. Frick, Corona - eine spirituelle Erfahrung?, 205.

16 Roser, Krankenhausseelsorge und Spiritual Care, 229.

17 Zit. n. Mertes, Keine Zeit für Kommentare, 321.

18 Mertes, Keine Zeit für Kommentare, 322. 
suchen, ihre Argumente zu hören und verstehen zu lernen - und schließlich anhand der Erfahrungen dieser Zeit den aktuellen und bleibenden Wert von Seelsorge (nicht nur in Corona-Zeiten, aber verdichtet für diese Zeit) herauszuarbeiten.

Verantwortungsbewusstsein kann sich auch darin zeigen, nicht jede Entscheidung der Klinikleitungen unhinterfragt hinzunehmen, als habe man ohnehin keine Wahl, vielleicht sogar froh zu sein darüber, dass einem die Entscheidung abgenommen wurde. Ist Schweigen dann wirklich Gold? Oder das Reden und Ringen um eine sachgemäße Güterabwägung, die umfassend die Positionen aller Betroffenen berücksichtigt, sich von diesen auch emphatisch beeindrucken lässt?

Es kann jetzt die Zeit sein, sich im gemeinsamen Schulterschluss von Teams und diözesanen Verantwortungsträgern aufzumachen. „Es gibt einen Ort jenseits von richtig und falsch. Dort treffen wir uns." Jetzt, da die erste Infektionswelle gemeistert ist, wäre es doch an der Zeit, ein Resümee zu ziehen und zu schauen, mit wem sich ein Treffen lohnen würde ...

\section{Literatur}

Ateliers et Presses de Taizé: Chants de Taizé, Freiburg i. Br.: Herder 2019.

Frick, Eckhard: Corona - eine spirituelle Erfahrung?, in: Spiritual Care 9/2 (2020) 205.

Kern, Karl: Mittwoch der Karwoche. Trauer - Grab (Leid - Liebe - Leben. Impulse zur Karwoche und Ostern 2020 am 8.4.2020). Dokument im Internet nicht mehr abrufbar; es liegt dem Verfasser vor.

Klessmann, Michael: Einleitung. Seelsorge in der Institution „Krankenhaus“, in: Klessmann, Michael: Handbuch der Krankenhausseelsorge, Göttingen: Vandenhoeck-Ruprecht ${ }^{2} 2002,13-27$.

Maio, Giovanni: Den kranken Menschen verstehen. Für eine Medizin der Zuwendung, Freiburg i. Br.: Herder 2015.

Mertes, Klaus: Keine Zeit für Kommentare, in: Stimmen der Zeit 145/5 (2020) 321-322.

Peng-Keller, Simon (Hg.): Gebet als Resonanzereignis. Annäherungen im Horizont von Spiritual Care, Göttingen: Vandenhoeck-Ruprecht 2017 (= Theologische Anstöße 7).

Roser, Traugott: Krankenhausseelsorge und Spiritual Care, in: Spiritual Care 6/2 (2017) 229-232.

Roser, Traugott: Seelsorge im Krankenhaus, in: Ders. (Hg.): Handbuch der Krankenhausseelsorge, Göttingen: Vandenhoeck-Ruprecht ${ }^{52019,13-33 .}$ 
Roser, Traugott/Peng-Keller, Simon/Kammerer, Thomas/Karle, Isolde/Lammer, Kerstin/Frick, Eckhard/Winiger, Fabian: Die Corona-Pandemie als Herausforderung für Spiritual Care. Handreichung für Seelsorger*innen, in: http://7b22a723 -d18b-4f61-9b60-a0e89eb41dca.filesusr.com/ugd/f9562a_a31ba104844340479cbe 4ce3b2821ed8.pdf [10.05.2020].

Vasseur, Clara/Bündgens, Johannes: Spiritualität der Wahrnehmung. Einführung und Einübung, Freiburg i. Br./München: Karl Alber 2015. 
